\title{
ON CERTAIN PROBLEMS OF APPROXIMATION IN THE COMPLEX DOMAIN*
}

\section{BY DUNHAM JACKSON}

When the paper with the above title was presented to the Society, I expected to publish it within a short time, and put on record at the moment only a brief abstract giving no very specific indication as to the results obtained. But the publication was delayed, and meanwhile J. L. Walsh, $†$ working quite independently, has treated problems of similar character by a different method, and has given a discussion which is in various ways considerably more thorough and comprehensive than that which I had contemplated, besides extending to other problems which I had not dealt with at all. Some of my conclusions nevertheless remain outside the scope of Walsh's article, and the purpose of the following paragraphs is to give an account of these results, with repetition of those stated by Walsh only so far as is necessary in order to exhibit the working of the method. The reader is referred to Walsh's paper also for detailed citations of the literature.

The problem of the present note is that of the convergence of a sequence of approximating polynomials for a function of a complex variable, the approximating polynomials being chosen so as to minimize an integral containing a power of the absolute value of the error. The method is an adaptation of one which has been applied to problems in the approximation of real functions, depending on Bernstein's theorem on the derivative of a real polynomial or of a trigonometric sum. A corresponding theorem for polynomials in the complex domain reads as follows: $\ddagger$

If $P_{n}(z)$ is a polynomial of the nth degree such that

$$
\left|P_{n}(z)\right| \leqq L \quad \text { for } \quad\left|z-z_{0}\right| \leqq R,
$$

* Presented to the Society, December 29, 1927.

$\dagger$ On the overconvergence of sequences of polynomials of best approximation, Transactions of this Society, vol. 32 (1930), pp. 794-816.

$\ddagger$ S. Bernstein, Leçons sur les Propriétés Extrémales et la Meilleure Approximation des Fonctions Analytiques d'une Variable Réelle, Paris, Gauthier-Villars, 1926, pp. 44-45. 
then

$$
\left|P_{n}^{\prime}(z)\right| \leqq n L / R \quad \text { for } \quad\left|z-z_{0}\right| \leqq R .
$$

The coefficients in $P_{n}(z)$ may be real or complex. The content of the assertion is of course unchanged if $\left|z-z_{0}\right| \leqq R$ is replaced by $\left|z-z_{0}\right|=R$ in the explicit statement.

Suppose now that $f(z)$ is a function analytic for $\left|z-z_{0}\right|<R$, and continuous on the circumference of the circle. Let a polynomial $P_{n}(z)$ of specified degree $n$ be chosen so as to minimize the integral

$$
\int_{C} \rho(s)\left|f(z)-P_{n}(z)\right|^{m} d s,
$$

the path of integration being the circumference, $s$ the arc length measured from a fixed point of the path, $\rho(s)$ a given nonnegative measurable function of $s$, for simplicity bounded and with a positive lower bound, and $m$ a given positive real number. It can be shown by well known methods that the minimum problem has at least one solution, and that for $m>1$ the solution is unique. Let $P_{n}(z)$ henceforth denote the particular polynomial, or a particular polynomial, which reduces the integral to its smallest value, and let $\gamma_{n}$ denote this smallest value.

Let $p_{n}(z)$ be an arbitrary polynomial of the $n$th degree; let $\epsilon_{n}$ be the maximum of $\left|f(z)-p_{n}(z)\right|$ for $\left|z-z_{0}\right|=R$; let $r_{n}(z)=f(z)-p_{n}(z)$; and let $\pi_{n}(z)=P_{n}(z)-p_{n}(z)$, so that

$$
\begin{gathered}
r_{n}(z)-\pi_{n}(z)=f(z)-P_{n}(z), \\
\gamma_{n}=\int_{C} \rho(s)\left|f(z)-P_{n}(z)\right|^{m} d s=\int_{C} \rho(s)\left|r_{n}(z)-\pi_{n}(z)\right|^{m} d s .
\end{gathered}
$$

Let $\mu_{n}$ be the maximum of $\left|\pi_{n}(z)\right|$ on the circumference, and $z_{1}$ a point of the circumference at which this value is taken on.

Throughout the circle, by the theorem of Bernstein cited above, $\left|\pi_{n}^{\prime}(z)\right| \leqq n \mu_{n} / R$, and

$$
\left|\pi_{n}(z)-\pi_{n}\left(z_{1}\right)\right| \leqq n \mu_{n}\left|z-z_{1}\right| / R
$$

in the part of this circle for which $\left|z-z_{1}\right| \leqq R /(2 n)$, and, in particular, on an arc of the circumference exceeding $R / n$ in length,

$$
\left|\pi_{n}(z)-\pi_{n}\left(z_{1}\right)\right| \leqq \mu_{n} / 2, \quad\left|\pi_{n}(z)\right| \geqq \mu_{n} / 2
$$


The reasoning is continued substantially as in similar situations elsewhere. ${ }^{*}$ Let $V$ and $v>0$ be upper and lower bounds for $\rho(s)$. If $\mu_{n} \geqq 4 \epsilon_{n}, \gamma_{n} \geqq(R v / n)\left(\mu_{n} / 4\right)^{m}$, while, on the other hand,

$$
\gamma_{n} \leqq \int_{C} \rho(s)\left|f(z)-p_{n}(z)\right|^{m} d s \leqq 2 \pi R V \epsilon_{n}^{m},
$$

and by combination of these relations

$$
\mu_{n} \leqq 4(2 \pi V / v)^{1 / m} n^{1 / m} \epsilon_{n} .
$$

Whether $\mu_{n} \geqq 4 \epsilon_{n}$ or not, we have

$$
\left|f(z)-P_{n}(z)\right|=\left|r_{n}(z)-\pi_{n}(z)\right| \leqq c n^{1 / m} \epsilon_{n},
$$

where $c$ is independent of $n$ and of the choice of $p_{n}(z)$ (and, incidentally, independent of $R$ ), the relation holding throughout the circle $\left|z-z_{0}\right| \leqq R$.

For convergence of $P_{n}(z)$ toward $f(z)$, uniformly throughout the circle, a sufficient condition therefore is that it be possible to choose polynomials $p_{n}(z)$ for each value of $n$ so that $\lim _{n \rightarrow \infty} n^{1 / m} \epsilon_{n}=0$. This condition is of course fulfilled if $f(z)$ is analytic on the circumference of the circle, as well as inside; but it is satisfied in other non-trivial cases also. For example, if $\left|a_{n}\right| \leqq A /\left(n^{p} R^{n}\right)$, $p>1$, in the power series development

$$
f(z)=a_{0}+a_{1}\left(z-z_{0}\right)+a_{2}\left(z-z_{0}\right)^{2}+\cdots,
$$

the first $n+1$ terms of the series give a polynomial $p_{n}(z)$ for which $\epsilon_{n}$ does not exceed a constant multiple of $(1 / n)^{p-1}$, and the condition for convergence is satisfied if $p>(1 / m)+1$, though $f(z)$ is certainly not analytic at all points of the circumference if there are numbers $B>0$ and $q$ such that $\left|a_{n}\right| \geqq B /\left(n^{q} R^{n}\right)$, the power series being divergent then for $\left|z-z_{0}\right|>R$.

If $f(z)$ is analytic for $\left|z-z_{0}\right|=R$, a corollary of the preceding work is what Walsh calls the overconvergence of the sequence $P_{n}(z)$ : if $R_{0}>R$ is the radius of convergence of the series (1), and if $R_{1}$ is any number $<R_{0}$, the sequence converges uniformly toward $f(z)$ for $\left|z-z_{0}\right| \leqq R_{1}$. Let it be supposed for definiteness that $R_{1}>R$, and let $R^{\prime}, R^{\prime \prime}$ be chosen so that

$$
R_{0}>R^{\prime}>R^{\prime \prime}>R_{1}>R \text {. }
$$

* See, for example, D. Jackson, The Theory of Approximation, New York, 1930, pp. 82-86, 96-98. 
The series (1) is convergent for $\left|z-z_{0}\right|=R^{\prime}$, and the general term $a_{n}\left(z-z_{0}\right)^{n}$ is bounded, so that $\left|a_{n}\right| \leqq c_{1} / R^{\prime n}$, where $c_{1}$, like each of the other quantities denoted below by the letter $c$ with a subscript, is independent of $n$. For $\left|z-z_{0}\right|=R$,

$$
\left|a_{n}\left(z-z_{0}\right)^{n}\right| \leqq c_{1}\left(R / R^{\prime}\right)^{n} ;
$$

if the sum of the first $n+1$ terms of the series is taken as $p_{n}(z)$,

$$
\begin{aligned}
\left|f(z)-p_{n}(z)\right| & =\left|a_{n+1}\left(z-z_{0}\right)^{n+1}+a_{n+2}\left(z-z_{0}\right)^{n+2}+\cdots\right| \\
& \leqq c_{1}\left[\left(\frac{R}{R^{\prime}}\right)^{n+1}+\left(\frac{R}{R^{\prime}}\right)^{n+2}+\cdots\right] \\
& =\frac{c_{1} R^{\prime}}{R^{\prime}-R}\left(\frac{R}{R^{\prime}}\right)^{n+1}=c_{2}\left(\frac{R}{R^{\prime}}\right)^{n+1} .
\end{aligned}
$$

The last member is an upper bound for $\epsilon_{n}$. Since $R^{\prime \prime}<R^{\prime}$, there is a $c_{3}$ such that

$$
n^{1 / m^{\prime}} \epsilon_{n} \leqq c_{2} n^{1 / m}\left(\frac{R^{\prime \prime}}{R^{\prime}}\right)^{n+1}\left(\frac{R}{R^{\prime \prime}}\right)^{n+1} \leqq c_{3}\left(\frac{R}{R^{\prime \prime}}\right)^{n+1} .
$$

Hence

$$
\begin{gathered}
\left|f(z)-P_{n}(z)\right| \leqq c c_{3}\left(\frac{R}{R^{\prime \prime}}\right)^{n+1}, \\
\left|f(z)-P_{n+1}(z)\right| \leqq c c_{3}\left(\frac{R}{R^{\prime \prime}}\right)^{n+2}<c c_{3}\left(\frac{R}{R^{\prime \prime}}\right)^{n+1}, \\
\left|P_{n+1}(z)-P_{n}(z)\right| \leqq 2 c c_{3}\left(\frac{R}{R^{\prime \prime}}\right)^{n+1}=c_{4}\left(\frac{R}{R^{\prime \prime}}\right)^{n+1} .
\end{gathered}
$$

Or, if $P_{n}(z)-P_{n-1}(z)$ is denoted by $Q_{n}(z)$, and the subscript in the last inequality is reduced by one unit accordingly,

$$
\left|Q_{n}(z)\right| \leqq c_{4}\left(\frac{R}{R^{\prime \prime}}\right)^{n} .
$$

Let

$$
Q_{n}(z)=\alpha_{0}+\alpha_{1}\left(z-z_{0}\right)+\cdots+\alpha_{n}\left(z-z_{0}\right)^{n} .
$$

Then

$$
\alpha_{k}=\frac{1}{2 \pi i} \int_{C} \frac{Q_{n}(z)}{\left(z-z_{0}\right)^{k+1}} d z
$$


On the path of integration, which is understood to be the circle of radius $R$, as before, $Q_{n}(z)$ is subject to (2), and consequently

$$
\left|\alpha_{k}\right| \leqq \frac{1}{2 \pi} \cdot 2 \pi R \cdot c_{4}\left(\frac{R}{R^{\prime \prime}}\right)^{n} \cdot\left(\frac{1}{R}\right)^{k+1}=c_{4}\left(\frac{R}{R^{\prime \prime}}\right)^{n}\left(\frac{1}{R}\right)^{k} .
$$

For $\left|z-z_{0}\right|=R_{1}$

$$
\left|\alpha_{k}\left(z-z_{0}\right)^{k}\right| \leqq c_{4}\left(\frac{R}{R^{\prime \prime}}\right)^{n}\left(\frac{R_{1}}{R}\right)^{k} \leqq c_{4}\left(\frac{R}{R^{\prime \prime}}\right)^{n}\left(\frac{R_{1}}{R}\right)^{n}=c_{4}\left(\frac{R_{1}}{R^{\prime \prime}}\right)^{n}
$$

Since $Q_{n}(z)$ is the sum of $n+1$ terms of the form $\alpha_{k}\left(z-z_{0}\right)^{k}$,

$$
\left|Q_{n}(z)\right| \leqq c_{4}(n+1)\left(\frac{R_{1}}{R^{\prime \prime}}\right)^{n}
$$

on the same circle, and as the right-hand member is the general term of a convergent series, the sequence $P_{n}(z)$, equivalent to the series $\sum Q_{n}(z)$, is uniformly convergent for $\left|z-z_{0}\right| \leqq R_{1}$.

This proof of overconvergence has been given merely for the sake of showing its relation to the method of the present note, without the use of conformal representation or Hölder's inequality; it is not comparable in generality with Walsh's treatment of the subject.

The proof of convergence throughout the given closed region, restricted above to the case of a circle, can be extended, and was extended in my original paper, to regions of somewhat more general shape. For the question of overconvergence in more general regions I am content to refer the reader to Walsh.

Let $C$ be a closed rectifiable curve, given by a pair of equations $x=\phi(s), y=\psi(s)$, where $s$ is the length of arc, and $\phi(l)=\phi(0), \psi(l)=\psi(0), l$ being the length of the curve, but $\left|\phi\left(s_{2}\right)-\phi\left(s_{1}\right)\right|+\left|\psi\left(s_{2}\right)-\psi\left(s_{1}\right)\right| \neq 0$ for $0 \leqq s_{1}<s_{2}<l$. Let it be supposed that $\phi^{\prime \prime}(s)$ and $\psi^{\prime \prime}(s)$ are defined at all points of the curve, and bounded. Let $S$ be the closed region having $C$ for its boundary. It is fairly apparent from the geometric significance of the hypotheses, and can be proved analytically by routine processes of some length and awkwardness but no particular difficulty, that there is a number $r_{0}>0$ such that at every point of $C$ a circle of radius $r_{0}$ can be drawn tangent to $C$, and containing in its interior and on its boundary only 
points belonging to $S$. The essential thing is that a single positive $r_{0}$ serves for all points of the curve uniformly.

Let $P_{n}(z)$ be a polynomial of the nth degree such that $\left|P_{n}(z)\right| \leqq L$ throughout $S$. If $Q$ is any specified point of $C$, there is a circle of radius $r_{0}$, having $Q$ on its circumference, and belonging wholly to $S$, a circle consequently throughout which the condition $\left|P_{n}(z)\right| \leqq L$ is satisfied. Hence, by the form of Bernstein's theorem previously cited, $\left|P_{n}^{\prime}(z)\right| \leqq n L / r_{0}$ at all points of the circumference, and at the point $Q$ in particular. Since $Q$ is an arbitrary point of $C$, it is recognized that $\left|P_{n}^{\prime}(z)\right| \leqq n L / r_{0}$ at all points of $C$, and consequently throughout the interior of $S$ also.

With the aid of this extension of Bernstein's theorem it is possible to carry over the earlier convergence proof to the case of a region satisfying the somewhat restrictive hypotheses that have been imposed on $S$. If $f(z)$ is analytic throughout the interior of $S$ and continuous on $C$, and if $P_{n}(z)$ is the polynomial, or a polynomial, of the nth degree, minimizing the integral

$$
\int_{C} \rho(s)\left|f(z)-P_{n}(z)\right|^{m} d s,
$$

the weight function $\rho(s)$ and the exponent $m$ having the same character as before, a sufficient condition for the uniform convergence of $P_{n}(z)$ toward $f(z)$ throughout the closed region $S$ is that polynomials $p_{n}(z)$ exist so that $\lim _{n \rightarrow \infty} n^{1 / m} \epsilon_{n}=0$, where $\epsilon_{n}$ is the maximum of $\left|f(z)-p_{n}(z)\right|$ on $C$.

The problem may be modified, in the case either of the circle or of the more general region $S$, by supposing that the integral to be minimized in defining $P_{n}(z)$ is an area integral extended over the region, instead of a contour integral. The convergence proof can be carried through essentially as before, except that the factor $n^{1 / m}$ in the statement of the sufficient condition for convergence is to be replaced by $n^{2 / m}$, because at the point where Bernstein's theorem enters into the proof an arc whose length is of the order of $1 / n$ has to be replaced by an area* having linear dimensions of the order of $1 / n$. More general problems, still accessible to the same method of treatment, could

* For comparison, see E. Carlson, On the convergence of trigonometric approximations for a function of two variables, this Bulletin, vol. 32 (1926), pp. 639-641. 
be set up by combining the integral around the boundary with integrals over specified curves interior to the region, or by using area integrals together with line integrals, or by admitting values of the error at isolated points in the expression to be minimized. It is clear that this type of generalization would lead ultimately to the consideration of a Stieltjes integral, though the precise degree of generality that would be practicable remains to be ascertained.

The University of Minnesota

\section{CONCERNING QUASI- $k$-FOLD TRANSITIVITY OF PERMUTATION GROUPS*}

BY R. D. CARMICHAEL

1. Introduction. Let $G$ denote a permutation group having the property that for every $l$ such that $1 \leqq l \leqq k$ it is true that when two sets of $l$ letters each are given, taken from the letters on which $G$ operates, then there exists in $G$ a permutation $P$ which transforms the first of these sets of letters in some order into the second. Then it will be said that $G$ is quasi- $k$-fold transitive. $\dagger$ It is clear that $G$ is transitive in the ordinary sense. Quasi- $k$-fold transitivity differs from $k$-fold transitivity in respect to the matter of order in the elements; in the latter a permutation $P$ exists when the order of the $l$ elements in each of the two sets is prescribed such that it transforms the one ordered set into the other; in the former we have to do with the transformations of unordered sets.

G. A. Miller $\ddagger$ has pointed out that, when $p$ is a prime number of the form $4 x+3$, the semi-metacyclic group of degree $p$ is quasi-2-fold transitive, in the sense of our definition, even though it is only singly transitive. In a paper not yet published Miller has easily proved that a quasi- $k$-fold transitive group is

* Presented to the Society, September 11, 1930.

† Since the text of this article was put into type Professor W. B. Carver has called my attention to a paper by W. B. Carver and Mrs. Estella Fisher King, this Bulletin, vol. 26 (1920), pp. 319-322, dealing with quasi- $k$-fold transitivity.

$\ddagger$ Transactions of this Society, vol. 28 (1926), p. 339. 